\title{
STRESS DISTRIBUTION AROUND A HOLE IN AN ELLIPSOIDAL SHELL*
}

$\mathrm{BY}$

\section{N. De SILVA And H. COHEN (University of Minnesota and Honeywell Research Center)}

Summary. This paper treats the stress distribution in an ellipsoidal shell of revolution with a hole. The problem is reduced to the solution of sets of eight simultaneous equations. If interest is restricted to the neighborhood of the hole, a simplification occurs requiring the solution of sets of four simultaneous equations.

1. Introduction. The problem of determining the stress concentration caused by holes in thin shells is a basic one which has not, however, been amenable to solution. The complicated differential equations governing the deformation have been the main source of trouble. Lur'e [1] gave a solution for the case of a hole in an infinitely long closed circular cylindrical shell using the equations of shallow shell theory. His solution was developed in terms of a "curvature" parameter and in the limiting case when the latter vanished, the solution reduced to the generalized plane stress distribution in the neighborhood of a hole in an infinite plate under bi-axial tension.

The present paper treats the stress distribution in an ellipsoidal shell of revolution with a circular hole the axis of which is parallel to the axis of revolution of the ellipsoid. The linear shallow shell theory of Green and Zerna [2] is suitably modified to constitute the framework of the problem. The method of solution is quite general and may be applied to any type support condition with an arbitrary surface loading. Specifically, in the interests of demonstrative results, the outer edge is assumed clamped and the loading uniform.

From the work of Reissner [3], a basic solution $\left\{S_{0}\right\}$ is given, which solves the problem for the domain without a hole. By an extension of the addition theorems for Bessel functions [4], $\left\{S_{0}\right\}$ is used as a guide to choose directly the solution $\{S\}$ which satisfies all the required mathematical conditions. The problem is reduced to solving sets of eight simultaneous algebraic equations.

Finally, if interest is restricted to the neighborhood of the hole, a residual solution $\left\{S_{d}\right\}$ is deduced such that the required solution is $\left\{S_{0}\right\}+\left\{S_{d}\right\}$. The problem now involves solving sets of four simultaneous equations.

2. The basic equations. Following Green and Zerna [2], the deformation of a shallow shell may be expressed in terms of a complex function $\psi$

$$
\psi=w+i K \phi,
$$

which satisfies the differential equation

$$
\left.e^{\alpha \beta} e^{\gamma \rho} \psi\right|_{\alpha \beta \gamma \rho}+\left.\left.\left(i / \lambda^{*}\right) \epsilon^{\alpha \rho} \epsilon^{\beta \gamma} z\right|_{\alpha \beta} \psi\right|_{\rho \gamma}=p / B
$$

where

$$
\lambda^{*}=\lambda /\left[12\left(1-\eta^{2}\right)\right]^{1 / 2}, \lambda=t / L, B=E \lambda^{3} L / 12\left(1-\eta^{2}\right) .
$$

*Received December 3, 1963; revised manuscript received October 16, 1964. The results presented in this paper were obtained in the course of research sponsored by the National Science Foundation under Research Grants NSF-G20192 and NSF-GK99. 
Here, $t$ is the thickness of the shell, $L$ is the smallest characteristic length of its middle surface $M, E, \eta$, are Young's modulus and Poisson's ratio respectively, $L z$ is the distance of $M$ from a base plane $P$, the bar before a subscript denotes covariant differentiation with respect to the metric tensor e of a $\xi_{\alpha}$ coordinate system defined in $P, p$ is related to $p_{(3)}$, the physical component of the load normal to $M$, by $L p_{(3)}=p$, and $\epsilon$ is the permutation tensor. In (2.1), $w$ is the component of the displacement vector perpendicular to $M, \phi$ is a function from which the membrane stress $\mathbf{n}$ is generated by:

$$
\begin{gathered}
n^{\alpha \beta}=\left.\epsilon^{\alpha \gamma} \epsilon^{\beta \rho} \phi\right|_{\gamma \rho}, \\
K=\left[12\left(1-\eta^{2}\right)\right]^{1 / 2} / \lambda^{2} E L .
\end{gathered}
$$

The couple tensor $\mathrm{m}$ and the shear stress vector $\mathrm{q}$ are given by

$$
m^{\alpha \beta}=-\left.B H^{\alpha \beta \rho \lambda} w\right|_{\rho \lambda} ; \quad q^{\alpha}=m^{\beta \alpha} / \beta,
$$

where

$$
H^{\alpha \beta \rho \lambda}=\frac{1}{2}\left\{e^{\alpha \lambda} e^{\beta \rho}+e^{\alpha \rho} e^{\beta \lambda}+\eta\left(\epsilon^{\alpha \lambda} \epsilon^{\beta \rho}+\epsilon^{\alpha \rho} \epsilon^{\beta \gamma}\right)\right\} .
$$

It is not too difficult to show [5] that for stress boundary conditions we specify, along a curve $C$ in $P$, the traction system $\{\sigma\}$ :

$$
\{\sigma\}=\left\{n^{\alpha \rho} u_{\alpha} ; \quad q^{\alpha} u_{\alpha}-\frac{\partial H}{\partial s} ; G\right\},
$$

where

$$
H=L \epsilon_{\beta \lambda} m^{\alpha \beta} u_{\alpha} u^{\lambda} ; \quad G=L m^{\alpha \beta} u_{\alpha} u_{\beta},
$$

$\mathbf{u}$ is the unit normal to $C$ and $\partial / \partial s$ is the derivative along the tangent to $C$. If we choose $C$ to be $\xi_{1}=$ constant, then

$$
\{\sigma\}=\left\{n_{(11)} ; n_{(12)} ; q_{(1)}-\frac{m_{(12) .2}}{2 \pi \xi_{1} R_{0}} ; m_{(11)}\right\},
$$

where the parentheses about the subscripts denote physical components, and comma denotes partial differentiation with respect to the $\xi_{\alpha}$ system in $P$, when the $\xi_{\alpha}$ are defined in terms of polar coordinates $(r, \theta)$ by

$$
\xi_{1}=r / R_{0}, \quad \xi_{2}=\theta / 2 \pi .
$$

Consider a shell with $M$ a surface of revolution obtained by rotating an ellipse about its semi-minor axis, Fig. 1. The surface $M$ is symmetric with respect to the axis of revolution $y$ and the closed curve which defines the edge line of $M$ in the base plane $P$. If $a$ and $b$ are the semi-major and semi-minor axes respectively and $r$ is distance parallel to the base plane from the axis of revolution to the points on $M$, we set $L=r_{0}$, where $r_{0}$ is the radius of the contour intersect of $M$ and $P$. In the base plane $P$, we consider polar coordinates $r, \theta$ with origin at the intersection of the axis of revolution of $M$ with $P$, and define non-dimensional coordinates $\xi_{\alpha}$

$$
\xi_{1}=r / r_{0} ; \quad \xi_{2}=\theta / 2 \pi .
$$

If $h$ is the height of the apex of $M$ above the base plane $P$, then by shallow shell approximation, 


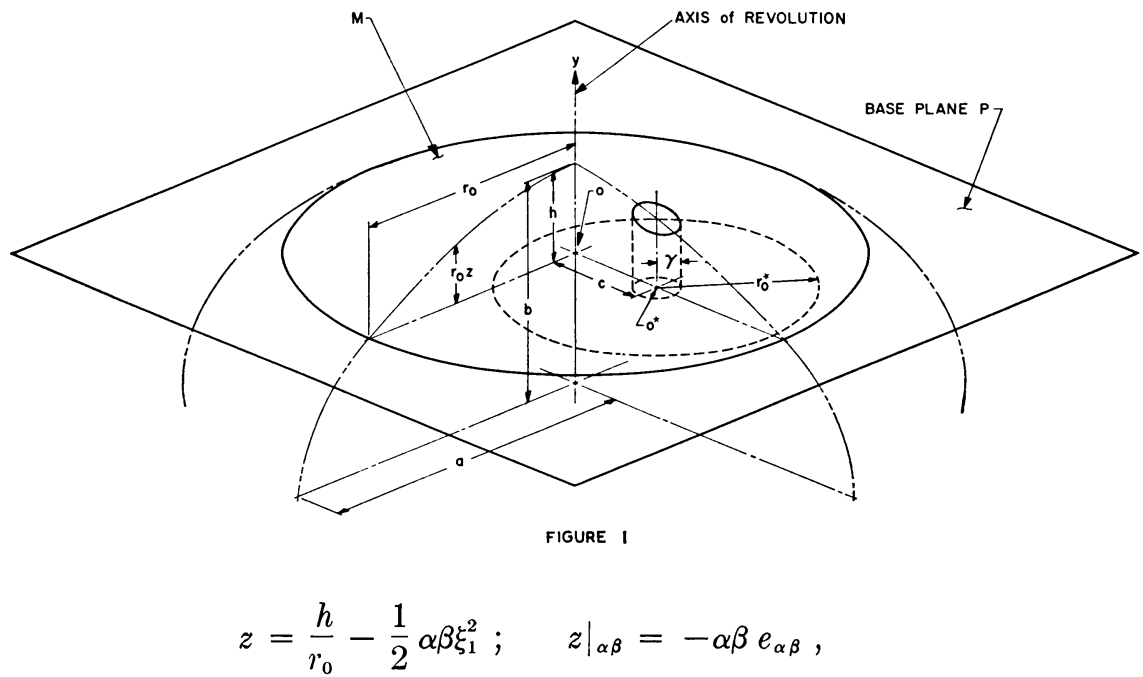

where $\alpha=b / a<1, \beta=r_{0} / a<1$. With the use of (2.10), Eq. (2.2) becomes:

$$
\nabla^{2} \nabla^{2} \psi-i \epsilon^{2} \nabla^{2} \psi=\frac{p}{B}
$$

where $\nabla^{2}$ is the Laplacian operator and

$$
\epsilon^{2}=\alpha \beta / \lambda^{*}
$$

The homogeneous equation corresponding to (2.11) has the general solution $\psi=\psi_{1}+\psi_{2}$ where

$$
\begin{aligned}
& \nabla^{2} \psi_{1}=0 \\
& \nabla^{2} \psi_{2}-i \epsilon^{2} \psi_{2}=0
\end{aligned}
$$

3. The basic solution: Uniformly loaded ellipsoidal shell. We consider a shallow ellipsoidal shell of revolution loaded symmetrically with respect to its axis. The basic homogeneous differential equations (2.13), (2.14) now involve derivatives with respect to $\xi_{1}$ only. In order to demonstrate the method of solution, we assume that $p$ is constant and that the edge is clamped. If $v_{\alpha}$ are the tangential components of middle surface displacement, the continuity and boundary conditions are:

at $\xi_{1}=0: n_{0(11)}, n_{0(22)}, m_{0(11)}, m_{0(22)}$, and $w_{0}$ are finite;

at $\xi_{1}=1$ (built-in-edge): $\quad v_{01}=w_{0}=w_{0,1}=0$.

From the general solution of (2.13) and (2.14), guided by the work of Reissner [3], and applying (3.1) and (2.1), we obtain:

$$
\begin{aligned}
& w_{0}=c_{1} \text { ber } \epsilon \xi_{1}-d_{1} \text { bei } \epsilon \xi_{1}+c_{3}, \\
& (K \phi)_{0}=d_{1} \text { ber } \epsilon \xi_{1}+c_{1} \text { bei } \epsilon \xi_{1}+p \xi_{1}^{2} / 4 B \epsilon^{2},
\end{aligned}
$$

where 


$$
\begin{aligned}
& c_{1}=(p / X)(\eta-1) \text { bei }^{\prime} \epsilon, \quad d_{1}=(p / X)(\eta-1) \text { ber }^{\prime} \epsilon \\
& c_{3}=(p / X)(\eta-1)\left[\text { bei } \epsilon \text { ber }^{\prime} \epsilon-\text { ber } \epsilon \text { bei }^{\prime} \epsilon\right], \\
& X=2 B \epsilon^{3}\left[\epsilon\left(\text { ber }^{\prime \prime} \epsilon \text { ber }^{\prime} \epsilon+\text { bei }^{\prime \prime} \epsilon \text { bei }^{\prime} \epsilon\right)-\eta\left(\text { ber }^{\prime 2} \epsilon+\text { bei }^{\prime 2} \epsilon\right)\right],
\end{aligned}
$$

and prime denotes differentiation with respect to the argument. From Eqs. (2.4), (2.6), (2.7) and (3.2), we compute the set of stresses $\left\{n_{0}^{\alpha \beta}, m_{0}^{\alpha \beta}, q_{0}^{\alpha}\right\}$.

In the base plane $P$ we consider a circle $C^{*}$ of radius $r_{0}^{*}$ and center located a distance $c$ from the origin $O$ such that $r_{0}^{*} \leq r_{0}-c$. We define non-dimensional variables $\theta_{\alpha}$ in terms of a new system of polar coordinates $\left(r^{*}, \theta^{*}\right)$, with pole $O^{*}$ at the center of the circle $C^{*}$ (Fig. 1), by

$$
\theta_{1}=r^{*} / r_{0}^{*}, \quad \theta_{2}=\theta^{*} / 2 \pi .
$$

The set $\left\{n_{0}^{\alpha \beta}, m_{0}^{\alpha \beta}, q_{0}^{\alpha}\right\}$ in the $\xi_{\alpha}$ system can now be transformed in the conventional manner into the set $\left\{N_{0}^{\alpha \beta}, M_{0}^{\alpha \beta}, Q_{0}^{\alpha}\right\}$ in the $\theta_{\alpha}$ system, represented by

$$
\begin{aligned}
& N_{0}^{11}=\frac{\epsilon}{K}\left[\frac { 1 } { \xi _ { 1 } ^ { 3 } } \{ \theta _ { 1 } ^ { 2 } + 2 \theta _ { 1 } ( \frac { c } { r _ { 0 } ^ { * } } ) \operatorname { c o s } 2 \pi \theta _ { 2 } + ( \frac { c } { r _ { 0 } ^ { * } } ) ^ { 2 } \operatorname { c o s } 4 \pi \theta _ { 2 } \} \left\{d_{1} \text { ber' }^{\prime} \xi_{1}\right.\right. \\
& \left.\left.+c_{1} \text { bei }^{\prime} \epsilon \xi_{1}\right\}+\frac{\epsilon}{\xi_{1}^{2}}\left(\frac{c}{r_{0}^{*}}\right)^{2} \sin ^{2} 2 \pi \theta_{2}\left\{c_{1} \text { ber } \epsilon \xi_{1}-d_{1} \text { bei } \epsilon \xi_{1}\right\}+\frac{p}{2 B \epsilon^{3}}\left(\frac{r_{0}}{r_{0}^{*}}\right)^{2}\right], \\
& \ldots \quad \\
& M_{0}^{11}=\frac{B \epsilon}{\xi_{1}^{3}}\left[( 1 - \eta ) ( c _ { 1 } \text { ber } ^ { \prime } \epsilon \xi _ { 1 } - d _ { 1 } \text { bei' } \epsilon \xi _ { 1 } ) \left\{\left(\theta_{1}+\frac{c}{r_{0}^{*}} \cos 2 \pi \theta_{2}\right)^{2}\right.\right. \\
& \left.-\left(\frac{c}{r_{0}^{*}}\right)^{2} \sin ^{2} 2 \pi \theta_{2}\right\}+\epsilon \xi_{1}\left(c_{1} \text { bei } \epsilon \xi_{1}+d_{1} \text { ber } \epsilon \xi_{1}\right) \\
& \times \\
& \left.\left.\times\left(\theta_{1}+\frac{c}{r_{0}^{*}} \cos 2 \pi \theta_{2}\right)^{2}+\eta\left(\frac{c}{r_{0}^{*}}\right)^{2} \sin ^{2} 2 \pi \theta_{2}\right\}\right] .
\end{aligned}
$$

Equation (3.5) defines the basic solution $\left\{S_{0}\right\}$. From Eqs. (2.8) and (3.5) we may determine $\left\{\sigma_{0}\right\}$ generated by $\left\{S_{0}\right\}$ across the curve $\theta_{1}=$ constant.

Applying the addition theorems for the Bessel functions $J_{0}(z), J_{0}^{\prime}(z)$ [4], corresponding theorems for ber $x$, $\operatorname{ber}^{\prime} x$, bei $x$, bei' $x$ are found to be

$$
\begin{aligned}
& \text { ber } \epsilon \xi_{1}=\sum_{n=0}^{\infty} A_{n} \cos n 2 \pi \theta_{2} ; \epsilon \xi_{1} \text { ber }^{\prime} \epsilon \xi_{1}=\sum_{n=0}^{\infty} C_{n} \cos n 2 \pi \theta_{2} ;
\end{aligned}
$$

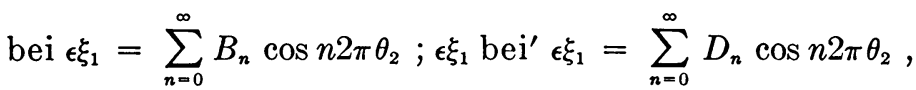

where at $\theta_{1}=1$

$$
\begin{aligned}
& A_{n}(1)=(-1)^{n} \epsilon_{n}\left[\operatorname{ber}_{n} \frac{\epsilon^{*} c}{r_{0}^{*}} \operatorname{ber}_{n} \epsilon^{*}-\operatorname{bei}_{n} \frac{\epsilon^{*} c}{r_{0}^{*}} \operatorname{bei}_{n} \epsilon^{*}\right], \\
& B_{n}(1)=(-1)^{n} \epsilon_{n}\left[\operatorname{ber}_{n} \frac{\epsilon^{*} c}{r_{0}^{*}} \operatorname{bei}_{n} \epsilon^{*}+\operatorname{bei}_{n} \frac{\epsilon^{*} c}{r_{0}^{*}} \operatorname{ber}_{n} \epsilon^{*}\right],
\end{aligned}
$$

with $\epsilon_{n}=1$ if $n=0$, and $\epsilon_{n}=2$ if $n>0$, 


$$
\begin{aligned}
& C_{n}(1)=\frac{(-1)^{n}}{2^{1 / 2}}\left[\epsilon ^ { * } \left\{\left(\operatorname{ber}_{n+1} \epsilon^{*}-\delta_{n} \operatorname{ber}_{n-1} \epsilon^{*}\right)\left(\operatorname{ber}_{n} \frac{\epsilon^{*} c}{r_{0}^{*}}+\operatorname{bei}_{n} \frac{\epsilon^{*} c}{r_{0}^{*}}\right)\right.\right. \\
& \left.+\left(\operatorname{bei}_{n+1} \epsilon^{*}-\delta_{n} \text { bei }_{n-1} \epsilon^{*}\right)\left(\operatorname{ber}_{n} \frac{\epsilon^{*} c}{r_{0}^{*}}-\operatorname{bei}_{n} \frac{\epsilon^{*} c}{r_{0}^{*}}\right)\right\}+\frac{\epsilon^{*} c}{r_{0}^{*}} \\
& \times\left\{\left(\operatorname{ber}_{n+1} \frac{\epsilon^{*} c}{r_{0}^{*}}-\delta_{n} \operatorname{ber}_{n-1} \frac{\epsilon^{*} c}{r_{0}^{*}}\right)\left(\operatorname{ber}_{n} \epsilon^{*}+\operatorname{bei}_{n} \epsilon^{*}\right)+\left(\operatorname{bei}_{n+1} \frac{\epsilon^{*} c}{r_{0}^{*}}\right.\right. \\
& \left.\left.\left.-\delta_{n} \operatorname{bei}_{n-1} \frac{\epsilon^{*} c}{r_{0}^{*}}\right)\left(\operatorname{ber}_{n} \epsilon^{*}-\operatorname{bei}_{n} \epsilon^{*}\right)\right\}\right] \text {, } \\
& D_{n}(1)=\frac{(-1)^{n}}{2^{1 / 2}}\left[\epsilon ^ { * } \left\{\left(\operatorname{ber}_{n+1} \epsilon^{*}-\delta_{n} \operatorname{ber}_{n-1} \epsilon^{*}\right)\left(\operatorname{bei}_{n} \frac{\epsilon^{*} c}{r_{0}^{*}}-\operatorname{ber}_{n} \frac{\epsilon^{*} c}{r_{0}^{*}}\right)\right.\right. \\
& \left.+\left(\operatorname{bei}_{n+1} \epsilon^{*}-\delta_{n} \operatorname{bei}_{n-1} \epsilon^{*}\right)\left(\operatorname{ber}_{n} \frac{\epsilon^{*} c}{r_{0}^{*}}+\operatorname{bei}_{n} \frac{\epsilon^{*} c}{r_{0}^{*}}\right)\right\}+\frac{\epsilon^{*} c}{r_{0}^{*}} \\
& \times\left\{\left(\operatorname{ber}_{n+1} \frac{\epsilon^{*} c}{r_{0}^{*}}-\delta_{n} \operatorname{ber}_{n-1} \frac{\epsilon^{*} c}{r_{0}^{*}}\right)\left(\text { bei }_{n} \epsilon^{*}-\text { ber }_{n} \epsilon^{*}\right)+\left(\text { bei }_{n+1} \frac{\epsilon^{*} c}{r_{0}^{*}}\right.\right. \\
& \left.\left.\left.-\delta_{n} \operatorname{bei}_{n-1} \frac{\epsilon^{*} c}{r_{0}^{*}}\right)\left(\operatorname{ber}_{n} \epsilon^{*}+\text { bei }_{n} \epsilon^{*}\right)\right\}\right]
\end{aligned}
$$

with $\delta_{n}=0$ if $n=0$, and $\delta_{n}=1$ if $n>0$. In (3.8) and (3.9),

$$
\epsilon^{*}=\left(r_{0}^{*} / r_{0}\right) \epsilon \text {. }
$$

Using the above addition theorems in (3.5) and neglecting $\left(c / r_{0}^{*}\right)^{2}$ compared to unity, the required physical components of the solution $\left\{S_{0}\right\}$ (1) take the form:

$$
\begin{aligned}
& N_{0(11)}=\frac{1}{K} \sum_{n=-3}^{\infty}\left[\left(\frac{r_{0}}{r_{0}^{*}}\right)^{2}\left\{E_{n}-\left(\frac{c}{r_{0}^{*}}\right)\left(E_{n-1}+E_{n+1}\right)\right\}-\frac{\epsilon^{2}}{2}\left(\frac{c}{r_{0}^{*}}\right)^{2}\right. \\
& \text {. } \left.\left\{F_{n}-\frac{1}{2}\left(F_{n-2}+F_{n+2}\right)-\frac{c}{2 r_{0}^{*}}\left(F_{n-1}+F_{n+1}\right)+\frac{c}{2 r_{0}^{*}}\left(F_{n-3}+F_{n+3}\right)\right\}\right] \\
& \cdot \cos n 2 \pi \theta_{2}+\frac{p}{2 B \epsilon^{2} K} \\
& N_{0(12)}=\frac{-1}{K}\left(\frac{c}{r_{0}^{*}}\right) \sum_{n=-2}^{\infty}\left[\left(\frac{r_{0}}{r_{0}^{*}}\right)^{2}\left\{\left(E_{n-1}-E_{n+1}\right)-\frac{3}{2}\left(\frac{c}{r_{0}^{*}}\right)\left(E_{n-2}-E_{n+2}\right)\right\}\right. \\
& \left.+\frac{\epsilon^{2}}{2}\left\{\left(F_{n-1}-F_{n+1}\right)-\frac{c}{2 r_{0}^{*}}\left(F_{n-2}-F_{n+2}\right)\right\}\right] \sin n 2 \pi \theta_{2}, \\
& M_{0(11)}=r_{0} B \sum_{n=-1}^{\infty}\left[\left(\frac{r_{0}}{r_{0}^{*}}\right)^{2}(1-\eta)\left\{G_{n}-\frac{c}{r_{0}^{*}}\left(G_{n-1}+G_{n+1}\right)\right\}+\epsilon^{2} H_{n}\right] \cos n 2 \pi \theta_{2}, \\
& M_{0(12)}=r_{0} B(1-\eta)\left(\frac{c}{r_{0}^{*}}\right) \sum_{n=-2}^{\infty}\left[( \frac { r _ { 0 } } { r _ { 0 } ^ { * } } ) ^ { 2 } \left\{\left(G_{n-1}-G_{n+1}\right)-\frac{3}{2}\left(\frac{c}{r_{0}^{*}}\right)\right.\right. \\
& \left.\left.\cdot\left(G_{n-2}-G_{n+2}\right)\right\}+\frac{\epsilon^{2}}{2}\left\{\left(H_{n-1}-H_{n+1}\right)-\frac{1}{2}\left(\frac{c}{r_{0}^{*}}\right)\left(H_{n-2}-H_{n+2}\right)\right\}\right] \\
& \cdot \sin n 2 \pi \theta_{2} \\
& Q_{0(1)}=B \epsilon^{2}\left(\frac{r_{0}}{r_{0}^{*}}\right) \sum_{n=-1}^{\infty}\left[E_{n}-\frac{c}{2 r_{0}^{*}}\left(E_{n-1}+E_{n+1}\right)\right] \cos n 2 \pi \theta_{2}
\end{aligned}
$$


where $E_{n}=d_{1} C_{n}+c_{1} D_{n}, F_{n}=d_{1} B_{n}-c_{1} A_{n}, G_{n}=c_{1} C_{n}-d_{1} D_{n}, H_{n}=c_{1} B_{n}+d_{1} A_{n}$, and $E_{n}=F_{n}=G_{n}=H_{n}=0$ if $n<0$.

4. Solution of ellipsoidal shell with a circular hole. We now consider the ellipsoidal shell to have a hole defined by the intersection $C_{M}^{*}$ of the middle surface $M$ with a circular cylindrical surface the axis of which is parallel to but not coincident with the axis of the ellipsoid, Fig. 1. The hole is stress free. The intersection $C_{M}^{*}$ in $M$ projects into a circle $C$ of radius $\gamma$ in the base plane $P$. The center of $C$ is located a distance $c$ from the axis in $P$. In the $\theta_{\alpha}$ system, the hole is defined by $\theta_{1} \leq \gamma / r_{0}^{*}=\gamma_{0}$. In a region exterior to the circle $C^{*}$ of radius $r_{0}^{*}$, the effect of the hole on the stress distribution is assumed negligible, appealing to the de Saint-Venant principle. The solution $\left\{S_{0}\right\}$ is therefore valid in this region and generates the system $\left\{\sigma_{0}\right\}\left(\theta_{1}=1\right)$ across the circle $C^{*}$. We consider next the region interior to the circle $C^{*}$, defined by $D: \gamma_{0} \leq \theta_{1} \leq 1,0 \leq \theta_{2} \leq 1$. In this region we seek a solution $\{S\}$ which will satisfy (2.11) and give rise to a system $\{\sigma\}$ such that

$$
\{\sigma\}\left(\gamma_{0}\right)=0 ; \quad\{\sigma\}(1)=\left\{\sigma_{0}\right\}(1) .
$$

It follows that $\{S\}$ stems from a complex function $\psi$ which satisfies Eqs. (2.11), (2.13) and (2.14). Guided by Eq. (3.10), and using (2.1), the appropriate solutions are

$$
w=\sum_{n=0}^{\infty} L_{n} \cos n 2 \pi \theta_{2} ; \quad K \phi=\sum_{n=0}^{\infty} M_{n} \cos n 2 \pi \theta_{2}+\frac{p^{*} \theta_{1}^{2}}{4 B \epsilon^{2}}\left(\frac{r_{0}^{*}}{r_{0}}\right)^{2},
$$

where

$$
\begin{gathered}
p^{*}=\left[1-\left(\gamma / r_{0}^{*}\right)^{2}\right]^{-1} p, \\
L_{0}=a_{10}+a_{50} \text { ber } \epsilon^{*} \theta_{1}-a_{60} \text { bei } \epsilon^{*} \theta_{1}+a_{70} \text { ker } \epsilon^{*} \theta_{1}-a_{80} \text { kei } \epsilon^{*} \theta_{1}, \\
M_{0}=a_{20}+a_{40} \log \theta_{1}+a_{60} \text { ber } \epsilon^{*} \theta_{1}+a_{50} \text { bei } \epsilon^{*} \theta_{1}+a_{80} \text { ker } \epsilon^{*} \theta_{1}+a_{70} \text { kei } \epsilon^{*} \theta_{1}
\end{gathered}
$$

and for $n>0$

$$
\begin{aligned}
& L_{n}=a_{1 n} \theta_{1}^{n}+a_{3 n} \theta_{1}^{-n}+a_{5 n} \operatorname{ber}_{n} \epsilon^{*} \theta_{1}-a_{6 n} \operatorname{bei}_{n} \epsilon^{*} \theta_{1}+a_{7 n} \operatorname{ker}_{n} \epsilon^{*} \theta_{1}-a_{8 n} \operatorname{kei}_{n} \epsilon^{*} \theta_{1}, \\
& M_{n}=a_{2 n} \theta_{1}^{n}+a_{4 n} \theta_{1}^{-n}+a_{6 n} \operatorname{ber}_{n} \epsilon^{*} \theta_{1}+a_{7 n} \operatorname{bei}_{n} \epsilon^{*} \theta_{1}+a_{8 n} \operatorname{ker}_{n} \epsilon^{*} \theta_{1}+a_{7 n} \operatorname{kei}_{n} \epsilon^{*} \theta_{1} .
\end{aligned}
$$

Note that $p^{*}$ is defined to ensure equilibrium of the shell. It follows that

$$
\begin{aligned}
& n_{(11)}=\frac{1}{K}\left(\frac{r_{0}}{r_{0}^{*}}\right)^{2} \sum_{n=0}^{\infty}\left[\frac{M_{n, 1}}{\theta_{1}}-\frac{n^{2}}{\theta_{1}^{2}} M_{n}\right] \cos n 2 \pi \theta_{2}+\frac{p^{*}}{2 K B \epsilon^{2}}, \\
& n_{(12)}=\frac{1}{K}\left(\frac{r_{0}}{r_{0}^{*}}\right)^{2} \sum_{n=0}^{\infty} n\left[\frac{M_{n, 1}}{\theta_{1}}-\frac{M_{n}}{\theta_{1}^{2}}\right] \sin n 2 \pi \theta_{2}, \\
& n_{(22)}=\frac{1}{K}\left(r_{0} / r_{0}^{*}\right)^{2} \sum_{n=0}^{\infty} M_{n, 11} \cos n 2 \pi \theta_{2}+\frac{p^{*}}{2 K B \epsilon^{2}}, \\
& m_{(11)}=-r_{0} B\left(\frac{r_{0}}{r_{0}^{*}}\right)^{2} \sum_{n=0}^{\infty}\left[L_{n, 11}+\eta\left\{\frac{L_{n, 1}}{\theta_{1}}-\frac{n^{2}}{\theta_{1}^{2}} L_{n}\right\}\right] \cos n 2 \pi \theta_{2}, \\
& m_{(12)}=r_{0} B\left(\frac{r_{0}}{r_{0}^{*}}\right)^{2}(1-\eta) \sum_{n=0}^{\infty} n\left[\frac{L_{n}}{\theta_{1}^{2}}-\frac{L_{n, 1}}{\theta_{1}}\right] \sin n 2 \pi \theta_{2},
\end{aligned}
$$




$$
\begin{aligned}
m_{(22)} & =r_{0} B\left(\frac{r_{0}}{r_{0}^{*}}\right)^{2} \sum_{n=0}^{\infty}\left[\eta L_{n, 11}+\frac{L_{n, 1}}{\theta_{1}}-\frac{n^{2} L_{n}}{\theta_{1}^{2}}\right] \cos n 2 \pi \theta_{2} \\
q_{(1)} & =B\left(r_{0} / r_{0}^{*}\right)^{3} \epsilon^{*^{3}} \sum_{n=0}^{\infty} N_{n} \cos n 2 \pi \theta_{2}
\end{aligned}
$$

where

$$
N_{n}=a_{6 n} \operatorname{ber}_{n}^{\prime} \epsilon^{*} \theta_{1}+a_{5 n} \operatorname{bei}_{n}^{\prime} \epsilon^{*} \theta_{1}+a_{8 n} \operatorname{ker}_{n}^{\prime} \epsilon^{*} \theta_{1}+a_{7 n} \mathrm{kei}_{n}^{\prime} \epsilon^{*} \theta_{1} .
$$

The coefficients $a_{m n}, m=1 \cdots 8$, can now be found by computing $\{\sigma\}$ from (2.8) and (4.5) and applying (4.1) with the use of (3.10). For any value of $n \geq 1, a_{m n}$ follows as the solution of an eighth order system of linear algebraic equations. For $n=0, m_{(12)}=$ $n_{(12)}=0$, and $q_{(1)}=0$ at $\gamma_{0}$ is satisfied identically; hence solution of a fifth order system is required with $a_{10}$ indeterminate.

5. Alternative solution: Residual problem. The order of the set of algebraic equations which yield the constants may be reduced by treating the problem as a residual one in which interest is restricted to the neighborhood of the hole. We redefine $\theta_{1}=r^{*} / \gamma$ such that the hole is specified by $\theta_{1} \leq 1$ and the region exterior to the hole is given by $1 \leq \theta_{1} \leq r_{0}^{*} / \gamma$. We now replace $\left(r_{0}^{*} / r_{0}\right),\left(c / r_{0}^{*}\right)$ by $\left(\gamma / r_{0}\right),(c / \gamma)$ respectively in our previous work.

In our addition theorem, noting that $\epsilon^{*} c / r_{0}^{*} \equiv \epsilon c / r_{0}$, we write

$$
\epsilon^{*}=\epsilon \gamma / r_{0}=\epsilon^{* *}(\gamma / c) ; \quad \epsilon\left(c / r_{0}\right)=\epsilon^{* *} .
$$

In (3.7) and (3.8), we, therefore, replace $\epsilon^{*}$ by $\epsilon^{* *}, c / r_{0}^{*}$ by $\gamma / c$. We now evaluate the set $\left\{N_{0(\alpha \beta)}, M_{0(\alpha \beta)}, Q_{0(\alpha)}\right\}$ at the boundary of the hole $\theta_{1}=1$. Neglecting $(\gamma / c)^{2}$ compared with unity, and applying the modified (3.7) and (3.8) to the modified (3.5), we obtain

$$
\begin{aligned}
N_{0(11)}(1)=\frac{1}{K} & \sum_{n=-3}^{\infty}\left\{\frac{1}{2}\left(\frac{r_{0}}{c}\right)^{2}\left[\left(E_{n-2}+E_{n+2}\right)-2\left(\frac{\gamma}{c}\right)\left(E_{n-3}+E_{n+3}\right)\right]\right. \\
& \left.-\frac{\epsilon^{2}}{2}\left[F_{n}-\frac{1}{2}\left(F_{n-2}+F_{n+2}\right)-\frac{1}{2}\left(\frac{\gamma}{c}\right)\left(F_{n-1}+F_{n+1}-F_{n-3}-F_{n+3}\right)\right]\right\} \\
& \cdot \cos n 2 \pi \theta_{2}+\frac{p}{2 B \epsilon^{2} K}, \\
N_{0(12)}(1)=- & \frac{1}{2 K} \sum_{n=-3}^{\infty}\left\{\left(\frac{r_{0}}{c}\right)^{2}\left[\left(E_{n-2}-E_{n+2}\right)-2\left(\frac{\gamma}{c}\right)\left(E_{n-3}-E_{n+3}\right)\right]\right. \\
& \left.+\frac{\epsilon^{2}}{2}\left[\left(F_{n-2}-F_{n+2}\right)+\left(\frac{\gamma}{c}\right)\left(F_{n-1}-F_{n+1}-F_{n-3}+F_{n+3}\right)\right]\right\} \sin n 2 \pi \theta_{2} \\
M_{0(11)}(1)=B r_{0} & \sum_{n=-3}^{\infty}\left\{( \frac { 1 - \eta } { 2 } ) ( \frac { r _ { 0 } } { c } ) ^ { 2 } \left[\left(G_{n-2}+G_{n+2}\right)+2\left(\frac{\gamma}{c}\right)\left(G_{n}-G_{n-1}-G_{n+1}+\frac{1}{2} G_{n-2}\right.\right.\right. \\
& \left.+\frac{1}{2} G_{n+2}-G_{n-3}-G_{n+3}\right)+\frac{\epsilon^{2}}{2}\left[(1+\eta) H_{n}+\left(\frac{1-\eta}{2}\right)\left(H_{n-2}+H_{n+2}\right)\right. \\
& \left.\left.+\left(\frac{1-\eta}{2}\right)\left(\frac{\gamma}{c}\right)\left(H_{n-1}+H_{n+1}-H_{n-3}-H_{n+3}\right)\right]\right\} \cos n 2 \pi \theta_{2},
\end{aligned}
$$




$$
\begin{aligned}
M_{0(12)}(1)= & \frac{B r_{0}(1-\eta)}{4} \sum_{n=-3}^{\infty}\left\{\left(\frac{r_{0}}{c}\right)^{2}\left[\left(G_{n-2}-G_{n+2}\right)-2\left(\frac{\gamma}{c}\right)\left(G_{n-3}-G_{n+3}\right)\right]\right. \\
& \left.+\epsilon^{2}\left[\left(H_{n-2}-H_{n+2}\right)+\left(\frac{\gamma}{c}\right)\left(H_{n-1}-H_{n+1}-H_{n-3}+H_{n+3}\right)\right]\right\} \sin n 2 \pi \theta_{2}, \\
Q_{0(1)}(1)= & \frac{B \epsilon^{2}}{2}\left(\frac{r_{0}}{c}\right) \sum_{n=-2}^{\infty}\left[\left(E_{n-1}+E_{n+1}\right)-\left(\frac{\gamma}{c}\right)\left(E_{n-2}+E_{n+2}\right)\right] \cos n 2 \pi \theta_{2} .
\end{aligned}
$$

Equations (5.2) yield the traction system $\left\{\sigma_{0}\right\}\left(\theta_{1}=1\right)$ generated by the basic solution $\left\{S_{0}\right\}$ at the hole. We seek a residual solution $\left\{S_{b}\right\}$ which satisfies (2.11) in $D$ and gives rise to a system $\left\{\sigma_{d}\right\}$ such that

$$
\left\{\sigma_{d}\right\}\left(\theta_{1}=1\right)=\left\{\sigma_{0}\right\}\left(\theta_{1}=1\right) ; \quad \lim _{\theta_{1} \rightarrow \infty}\left\{\sigma_{d}\right\}=0 .
$$

The second of (5.3) requires that we discard in $\psi_{1}$ solutions $\theta_{1}^{n}$ and in $\psi_{2}$ solutions $H_{n}^{(2)}(z)$. Hence in (4.3) we set $a_{50}=a_{60}=0$, in (4.4) $a_{1 n}=a_{2 n}=a_{5 n}=a_{6 n}=0$, and modify these expressions by replacing $\epsilon^{*}$ by $\epsilon^{* *} \gamma / c$. Similarly, we modify (4.2) and (4.5) by replacing $r_{0}^{*}$ by $\gamma, \epsilon^{*}$ by $\epsilon^{* *} \gamma / c$. For $n \geq 1$, the coefficients $a_{m n}, m=1,2,3,4$ are found, on applying $(5.3)_{1}$, by solving a fourth order system of linear equations. For $n=0$, there are three constants to be determined by specifying $n_{d(11)}, m_{d(11)}, q_{d(1)}$. The required solution is then $\{S\}=\left\{S_{0}\right\}-\left\{S_{d}\right\}$.

6. Some numerical results and discussion. The solution of the residual problem treated in the previous section was applied to obtain the stress distribution in the neighborhood of a hole with $\gamma / r_{0}=0.1$. Two other parameters were also specified: $\epsilon$, a measure of the shallowness of the shell (as $\epsilon$ approaches zero, we have plate behavior) and $c / \gamma$, a measure of the location of the hole relative to the axis of revolution. Two values of each were chosen: $\epsilon=1.1,4.0 ; c / \gamma=0,3$. In all cases, then, the hole is located away from the boundary layer. The value of 0.3 was assigned to $\eta$. The following dimensionless stresses were defined:

\begin{tabular}{|c|c|c|c|c|c|c|}
\hline \multirow[t]{2}{*}{$\theta^{*}$} & \multicolumn{2}{|c|}{$n_{(22)}^{*} \times 10^{2}$} & \multicolumn{2}{|c|}{$m_{(22)}^{*} \times 10^{2}$} & \multicolumn{2}{|c|}{$q_{(2)}^{*} \times 10^{2}$} \\
\hline & $c / \gamma=3$ & $c / \gamma=0$ & $c / \gamma=3$ & $c / \gamma=0$ & $c / \gamma=3$ & $c / \gamma=0$ \\
\hline 0 & 17.882 & 21.340 & -1.6381 & -1.6402 & 0.71275 & 0.0 \\
\hline$\pi / 4$ & 18.826 & 21.340 & -1.5910 & -1.6402 & 0.72150 & 0.0 \\
\hline$\pi / 2$ & 19.538 & 21.340 & -1.6054 & -1.6402 & 0.26719 & 0.0 \\
\hline $3 \pi / 4$ & 20.313 & 21.340 & -1.6207 & -1.6402 & -0.09751 & 0.0 \\
\hline$\pi$ & 20.875 & 21.340 & -1.6721 & -1.6402 & -0.19281 & 0.0 \\
\hline
\end{tabular}

$$
n_{(\alpha \beta)}^{*}=\frac{\left(t / r_{0}\right) n_{(\alpha \beta)}}{r_{0} p_{(3)}}, m_{(\alpha \beta)}^{*}=\frac{m_{(\alpha \beta)}}{r_{0}^{2} p_{(3)}}, q_{(\alpha)}^{*}=q_{(\alpha)} / r_{0} p_{(3)} .
$$

Tables 1 and 2 give the values of $n_{(22)}^{*}, m_{(22)}^{*}$ and $q_{(2)}^{*}$ at the edge of the hole $\theta_{1}=1.0$,

TABLE 1. $\epsilon=4, \gamma / r_{0}=0.1$ Values of Nonvanishing Stresses at the Boundary of the Hole, $r^{*} / \gamma=1$ 
TABle 2. $\epsilon=1.1, \gamma / r_{0}=0.1$ Values of Nonvanishing Stresses at the Boundary of the Hole, $r^{*} / \gamma=1$

\begin{tabular}{|c|c|c|c|c|c|c|}
\hline \multirow[t]{2}{*}{$\theta^{*}$} & \multicolumn{2}{|c|}{$n_{(22)}^{*} \times 10^{2}$} & \multicolumn{2}{|c|}{$m_{(22)}^{*} \times 10^{2}$} & \multicolumn{2}{|c|}{$q_{(2)}^{*} \times 10^{2}$} \\
\hline & $c / \gamma=3$ & $c / \gamma=0$ & $c / \gamma=3$ & $c / \gamma=0$ & $c / \gamma=3$ & $c / \gamma=0$ \\
\hline 0 & 9.2014 & 10.972 & -10.687 & -14.090 & 19.709 & 0.0 \\
\hline$\pi / 4$ & 9.2663 & 10.972 & -11.036 & -14.090 & 25.255 & 0.0 \\
\hline$\pi / 2$ & 9.6251 & 10.972 & -11.649 & -14.090 & 19.161 & 0.0 \\
\hline$\pi / 4$ & 10.647 & 10.972 & -12.227 & -14.090 & 15.791 & 0.0 \\
\hline$\pi$ & 10.677 & 10.972 & -13.581 & -14.090 & 9.837 & 0.0 \\
\hline
\end{tabular}

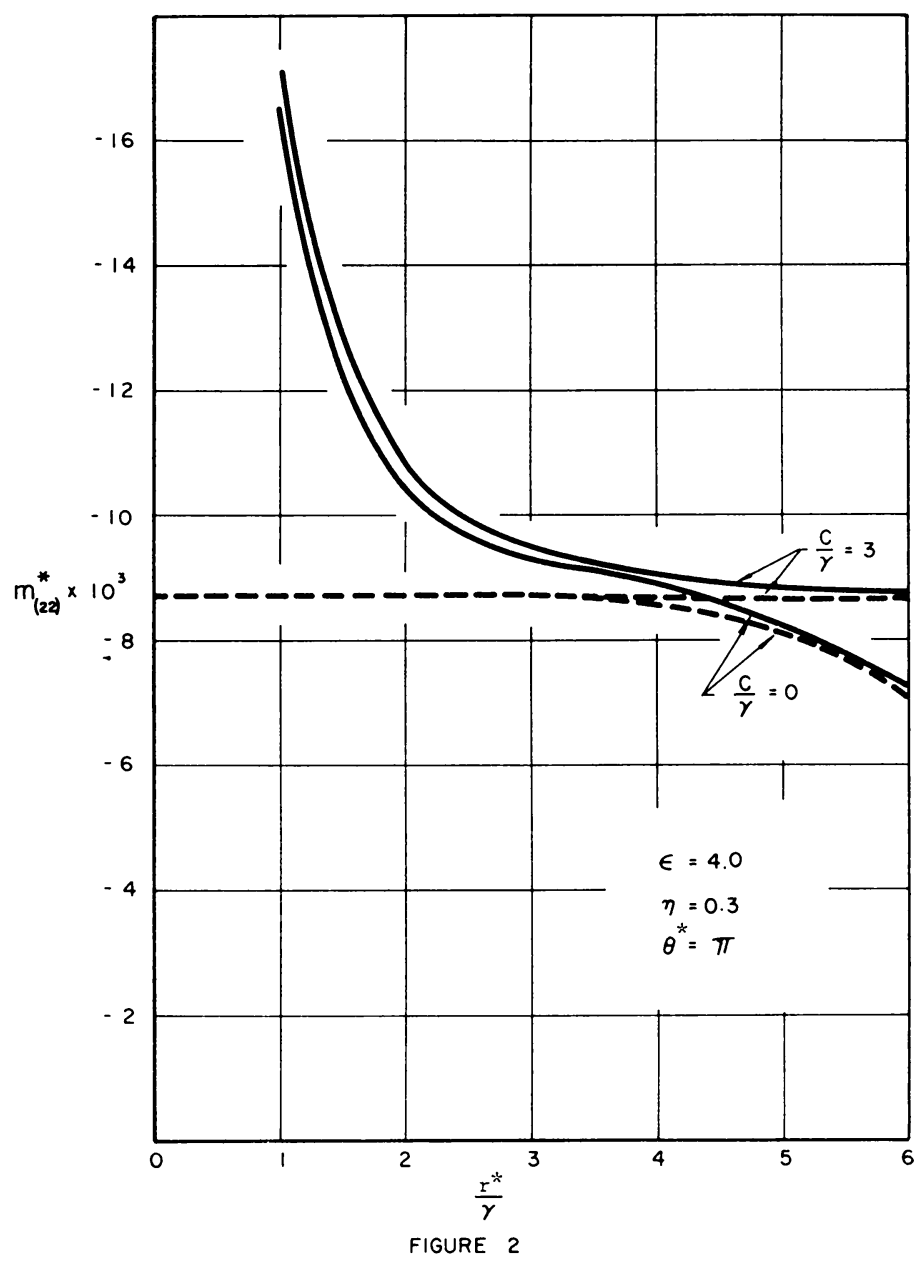


when $\epsilon=4$ and 1.1 respectively. In Figs. 2 and 3, $m_{(22)}^{*}$ and $n_{(22)}^{*}$ are plotted as functions of $r^{*} / \gamma$ for $\epsilon=4, \theta^{*}=\pi$. The dashed curve gives the variation when there is no hole. These stresses have a maximum value at the edge of the hole and become asymptotic to the respective no-hole solutions. A similar behavior will occur for $\epsilon=1.1$

We use the value at the apex of the basic solution as a reference to define stress concentration factors $k_{b}, k_{m}$ :

We obtain

$$
\begin{aligned}
& \left\{k_{b}, k_{m}\right\}=\left\{\frac{m_{(22)}^{*}(1, \pi)}{m_{0(22)}^{*}(0)}, \frac{n_{(22)}^{*},(1, \pi)}{n_{(22)}^{*}(0)}\right\} \text { for } \frac{c}{\gamma}=0, \\
& \left\{k_{b}, k_{m}\right\}=\frac{m_{(22)}^{*}(1, \pi)}{m_{0(22)}^{*}(3, \pi)}, \frac{n_{(22)}^{*},(1, \pi)}{n_{0(22)}^{*}(3, \pi)} \quad \text { for } \frac{c}{\gamma}=3 .
\end{aligned}
$$

\begin{tabular}{c|c|c|c|c}
$\epsilon$ & \multicolumn{2}{|c|}{$k_{b}$} & \multicolumn{2}{|c}{$k_{m}$} \\
\hline & $c / \gamma=3$ & $c / \gamma=0$ & $c / \gamma=3$ & $c / \gamma=0$ \\
4.0 & 1.941 & 1.904 & 1.952 & 1.996 \\
1.1 & 1.723 & 1.787 & 2.167 & 2.227
\end{tabular}

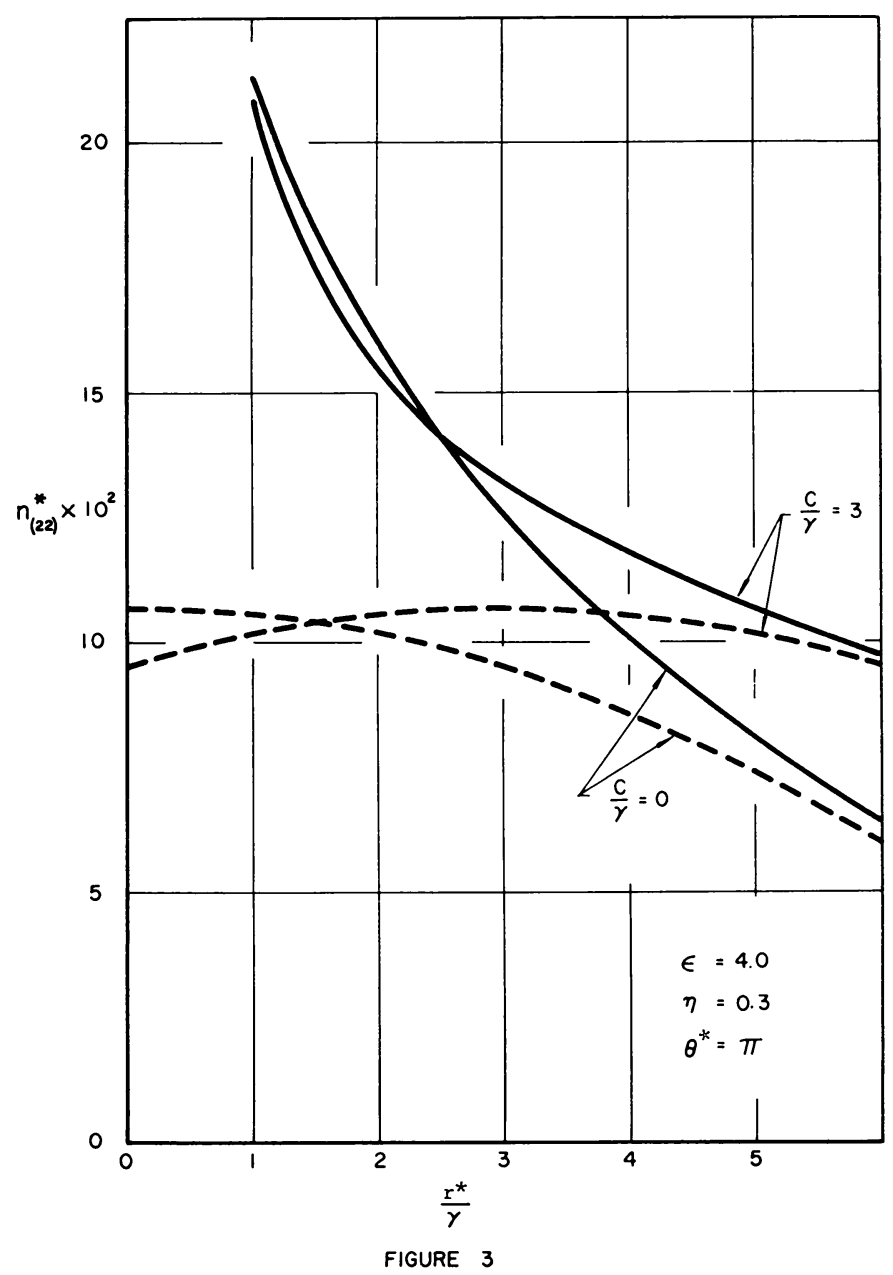


Increasing $c / \gamma$ from 0 to 3 results in decreased values of $k_{b}$ and $k_{m}$ for a particular value of $\epsilon$. An exception occurred for $k_{b}$ when $\epsilon=4$. A re-examination of the basic solution $\left\{S_{0}\right\}$ showed that $m_{0(22)}^{*}$ had a wave behavior near the apex and that its greatest absolute value occurred nearer the edge of the hole than the apex. This would explain the increase of $k_{b}$ in this case. Finally, we note that the bending factor, $k_{b}$, increases as $\epsilon$ increases, although the magnitude of the bending stresses decreases. Similarly, the membrane factor, $k_{m}$, increases as $\epsilon$ decreases, although the magnitude of the membrane stresses decreases.

\section{References}

1. A. I. Lur'e, Prikl. Mat. Mekh, 10 (1946) 397-406.

2. A. E. Green and W. Zerna, Theoretical Elasticity, Oxford University Press, London (1954)

3. E. Reissner, J. Math. Phys. 25 (1947) 279-300

4. G. N. Watson, Theory of Bessel Functions, Cambridge University Press, London, 1952

5. C. N. DeSilva and H. Cohen, University of Minnesota Institute of Technology Report NSF G-20192, September 1963 